\title{
Approaches of Turkish Dentists in Cases of Orthodontic Lingual Retainer Failures
}

\author{
Abdurahman Küçükönder ${ }^{1}$ iD, Ömer Hatipoğlu² (D) \\ Private practice, Kahramanmaras, Turkey \\ 2Department of Restorative Dentistry, Sutcu Imam University, Kahramanmaras, Turkey
}

Cite this article as: Küçükönder A, Hatipoğlu Ö. Approaches of Turkish Dentists in Cases of Orthodontic Lingual Retainer Failures. Turk J Orthod 2020; 33(4): $239-45$

Main points:

- Fixed lingual retainers are used to prevent relapse after orthodontic treatments. However, failure of lingual retainers may occur during retention period.

- Turkish dentists generally refer patients to orthodontists in cases of lingual retainer failure.

- In terms of the factors affecting the choice to remove a failed bonded retainer among dentists, the orthodontist's opinion appears to be the most important whereas the patient's demand is the least important factor.

\section{ABSTRACT}

Objective: This study aimed to investigate the approaches of Turkish dentists in cases of orthodontic lingual retainer failures.

Methods: A self-administered questionnaire was used to quantify dentists' approaches to lingual retainer failures. The first part of the study investigated the demographic characteristics. In the second part, dentists' approaches to cases of failed retainers were assessed. The third part had questions related to the type of retainers bonded solely to the canines or to all the 6 anterior teeth. Descriptive statistics were done with Pearson's $X 2$ test, and Mann-Whitney $U$ test was used.

Results: A total of 320 Turkish dentists participated in the survey. Experienced and public dentists preferred to advise the patients whose retainers had failed to contact their orthodontist more frequently $(p<0.05)$. Regarding their approach to patients who requested removal of the bonded retainer, inexperienced dentists more frequently preferred to refer the patients to an orthodontist $(p<0.05)$. With regard to factors affecting the choice to remove a bonded retainer, the most and the least importance were attributed to the orthodontist's opinion and the patient's demand, respectively.

Conclusion: Turkish dentists prefer referring their patients to orthodontists rather than performing procedures in cases of failure associated with bonded retainers. Different demographic characteristics seem to have an impact on these approaches.

Keywords: Orthodontic retainer, retention, survey

\section{INTRODUCTION}

After orthodontic treatment, despite successful treatment process, the teeth tend to return to their initial position, and this is known as relapse. Relapse, which is observed in most patients, is usually caused by stretching of the periodontal fibers (1). Retention after orthodontic treatment is a process that is performed almost regularly to prevent relapse (2). Retention is an indispensable requirement to succeed in orthodontic treatment and prevent occlusions from returning to the pre-treatment positions (3).

In almost every patient, orthodontic retention is used to stabilize the treatment results and prevent dental changes after treatment (4). Retention procedures vary from country to country. For instance, in the Netherlands, Norway, the United States, and Australia, bonded retainers are preferred more frequently in the mandibular region, whereas in the United Kingdom, removable retention is more frequently preferred in both the maxillary and mandibular regions (5-9). In a study conducted in 2016, Turkish orthodontists reported that they commonly used bonded retainers as the retention protocol in both maxillary and mandibular regions (10). Rigid canine-to-canine bonded retainers are attached solely to the canines (3-3 retainers), although they may also be 
bonded to all the 6 anterior teeth (3-2-1-1-2-3 retainers), and 3-21-1-2-3 lingual retainers are generally considered to be more effective in maintaining the position of the anterior teeth $(11,12)$.

However, lingual retainers may cause some problems over time as they stay in the mouth for a long period. Using long-term lingual retainers may increase plaque accumulation and gingival recession (13). In addition, because the lingual region is more exposed to the forces of mastication, the failure rate is higher in this region (5). Some studies reported that retainers often failed within a few months after bonding $(14,15)$. In the early stages of retainer administration, follow-up is performed mainly by the orthodontist, and in the following periods, patients are usually referred to general practitioners (16).

Dentists may have different approaches regarding lingual retainer failure cases due to the differences in the demographic characteristics. To date, to the best of our knowledge, only one study was conducted in relation to this topic in Switzerland (16), and the perspectives of Turkish dentists have not yet been investigated. The purpose of this study was to investigate the approaches of Turkish dentists in lingual retainer failure cases.

\section{METHODS}

Ethical approval was obtained from the ethics committee at Sutcu Imam University in Turkey (approval no. 2019-66). Written informed consent was obtained from the dentists who participated in the survey. The sample size was calculated using the Raosoft web survey software (http://www.raosoft.com/samplesize.html). With a $90 \%$ confidence level, $5 \%$ alpha error, 50\% response distribution rate, and 26,674 population size (the number of dentists in Turkey according to TUIK statistical data), a total of 268 participants were required (17). In February 2019, a link that directed people to a web-based questionnaire page (Google forms) was sent to the Turkish dentists via a web platform.

\section{Table 1. Questionnaire used for the study}

1. What is your sex?

a) Male

b) Female

2. How much experience do you have in the profession?

a) $\leq 10$ years

b) $>10$ years

3. Do you work at a private institution or a public institution?

a) Private

b) Public

4. Which option is your approach to patients whose retainer failed (debonded or broken)?

a) I advise patients to contact their orthodontists (A1)

b) I remove the retainer completely and I do not bond the retainer again (A2)

c) I bond the retainer again (A3)

d) I change the failed retainer with a new one (A4)

5. Which option is your approach to patients who request removal of a bonded retainer?

a) I explain to the patients all the possible consequences, and then I remove the retainer ( $A 1)$

b) I explain to the patients all the possible consequences, and I do not remove the retainer (A2)

c) I refer the patients to the orthodontists who bonded the retainer (A3)

6. How much is the factor of time of retention effective in your decision to remove the retainer (score between 0 and 5 )?

7. How much is the factor of patient's demand effective in your decision to remove the retainer (score between 0 and 5)?

8. How much is the factor of orthodontist's opinion effective in your decision to remove the retainer (score between 0 and 5 )?

9. How much is the factor of periodontal status effective in your decision to remove the retainer (score between 0 and 5 )?

10. How much is the factor of need for a new restoration effective in your decision to remove the retainer (score between 0 and 5 )?

11. What do you recommend to the patients with retainers for oral hygiene? (Multiple options can be checked)

a) Same as a normal patient

b) Interdental brush

c) Dental floss

d) Toothpick

e) Others

12. Which type of retainer (3-3 or 3-2-1-1-2-3) retains teeth efficiently?

13. Which type of retainer (3-3 or 3-2-1-1-2-3) complicates oral hygiene more?

14. Which type of retainer (3-3 or 3-2-1-1-2-3) prevents restoring teeth more?

15. Which type of retainer (3-3 or 3-2-1-1-2-3) increases periodontal problems more? 
The questionnaire was prepared on the basis of a previous survey study (16). The first part of the questionnaire included questions about demographic characteristics such as sex, experience, and workplace of the participants. The second part investigated the approaches of the participants to patients whose lingual retainers had failed (debonded or broken) and who referred to the dentists to remove the retainer. In addition, the effects of factors such as time of retention, the patient's demand, the orthodontist's opinion, periodontal status, and the need for a new restoration on the decision to remove the retainer were investigated (scores between 0 and 5). Furthermore, the questionnaire included questions about oral hygiene recommendations for patients with fixed retainers. The third part included questions about the efficiency of each type of retainer (3-3 or 3-2-1-1-2-3). The survey questions are presented in Table 1.

\begin{tabular}{|c|c|c|c|}
\hline $\begin{array}{l}\text { Demographic } \\
\text { characteristics }\end{array}$ & Factors & $\mathbf{n}$ & $\%$ \\
\hline \multirow[t]{2}{*}{ Sex } & Male & 126 & 39.4 \\
\hline & Female & 194 & 60.6 \\
\hline \multirow[t]{2}{*}{ Experience } & $\leq 10$ years & 260 & 81.2 \\
\hline & $>10$ years & 60 & 18.8 \\
\hline \multirow[t]{2}{*}{ Workplace } & Private & 172 & 53.8 \\
\hline & Public & 148 & 46.2 \\
\hline
\end{tabular}

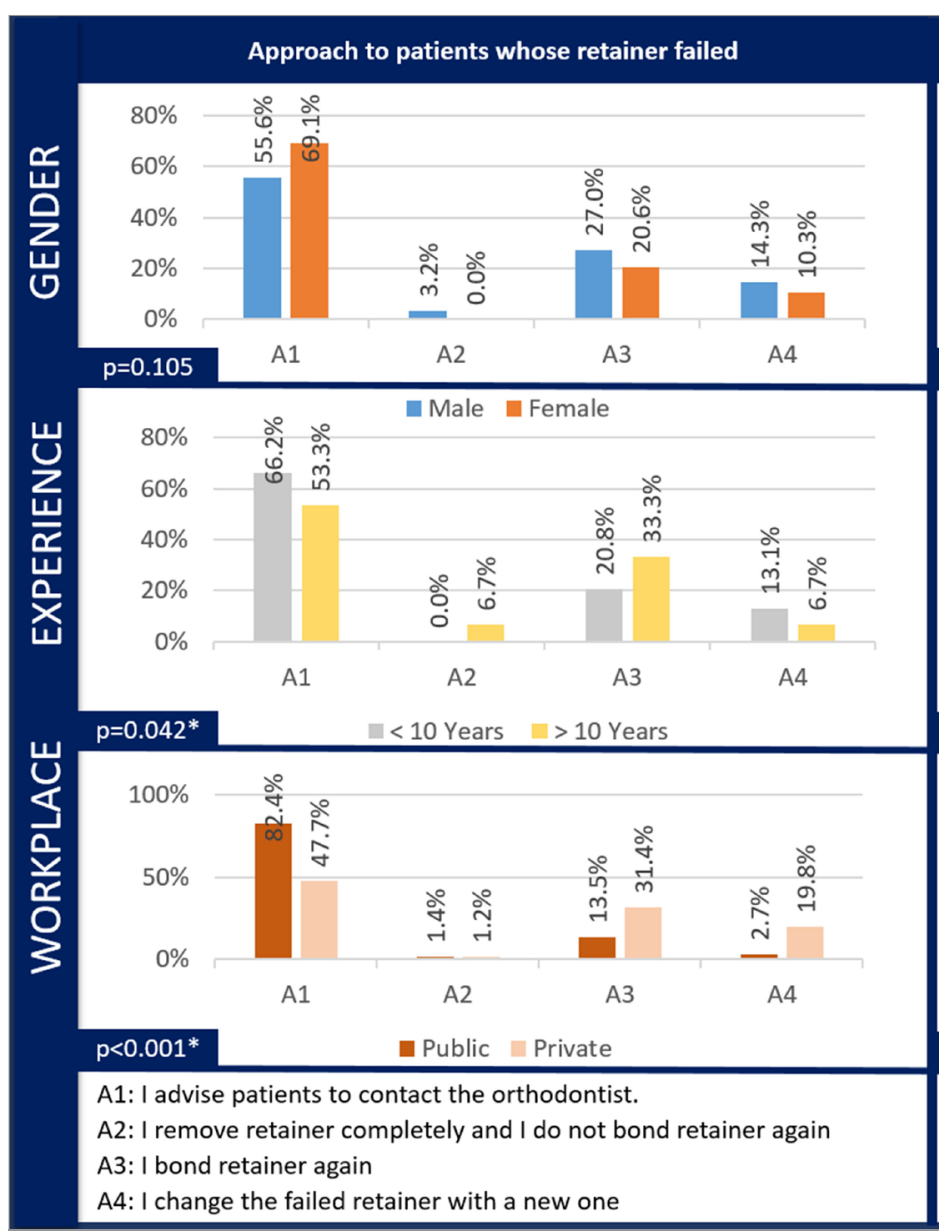

\section{Statistical Analysis}

Data analysis was performed using the Statistical Package for Social Sciences version 23.0 software (IBM Corp.; Armonk, NY, USA). As the data were not normally distributed, the mean values of the factors that affected the participants' choice to remove a bonded retainer were compared on the basis of demographic characteristics using (nonparametric) Mann-Whitney $U$ test. Answers related to types of retainers and approaches of Turkish dentistry practitioners were analyzed using a $x^{2}$ test with descriptive statistics. The Cronbach's alpha internal consistency coefficient was determined to verify the reliability of the survey. The probability level for statistical significance was set at $a=0.05$.

\section{RESULTS}

A total of 320 Turkish dentists participated in the survey. The distribution of the participants $(n=320)$ according to their sex (male or female), experience ( $\leq 10$ years or $>10$ years), and workplace (public or private) is shown in Table 2. A total of $60.6 \%$ of the participants were women, $81.2 \%$ had $\leq 10$ years of experience, and $53.8 \%$ worked at private clinics (Table 2). The survey had an adequate reliability with a Cronbach's alpha coefficient of 0.723 .

Most of the participants preferred to advise the patients whose retainers failed to contact an orthodontist. However, very few participants preferred to remove the retainer and did not bond the retainer again (Figure 1). The participants with an experience

\section{Approach to patients who request removel of a bonded retainer}

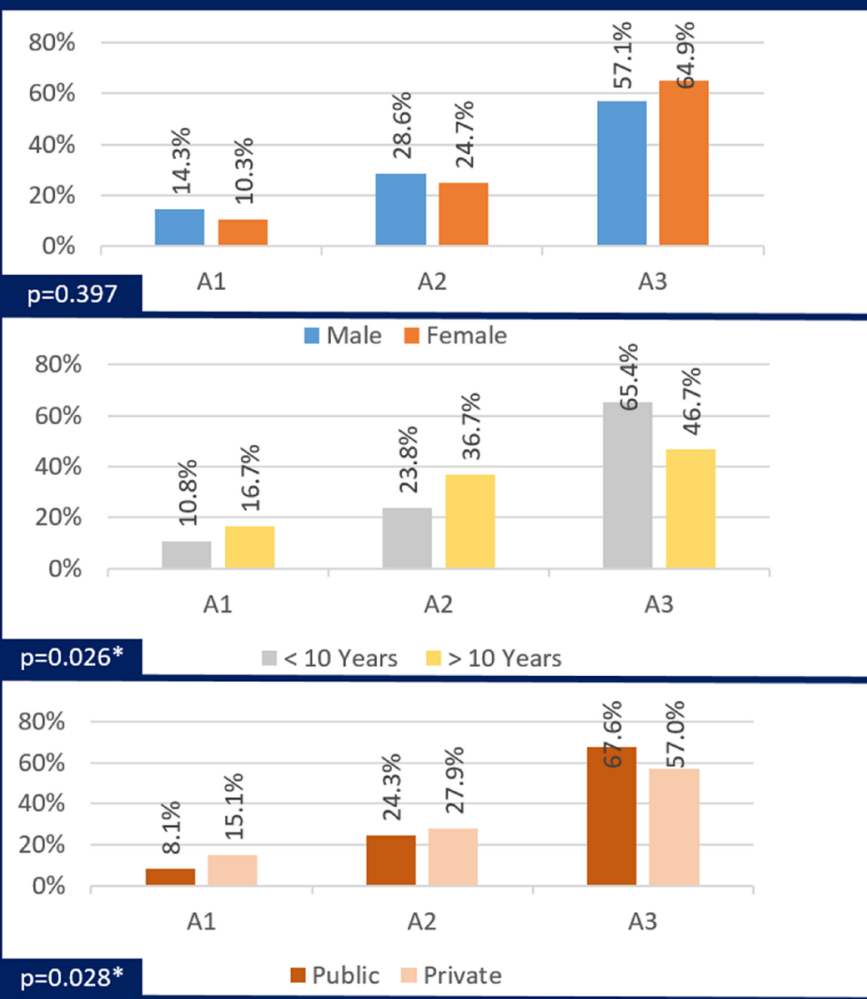

A1: I describe to patients all the possible results, and I remove the retainer A2: I describe to patients all the possible results, and I do not remove the retainer

A3: I refer patients to orthodontists who bonded the retainer previously 
Table 3. Statistical data comparing the participants' demographic characteristics and factors affecting their choice of removing a bonded retainer using Mann-Whitney U test.

\begin{tabular}{|c|c|c|c|c|c|}
\hline \multirow[b]{2}{*}{ Demographic characteristics } & \multirow{2}{*}{$\begin{array}{l}\text { Factors affecting the choice of } \\
\text { removing a bonded retainer }\end{array}$} & \multicolumn{4}{|c|}{ Dentists' scores } \\
\hline & & Mean $\pm S D(F 1)$ & Mean $\pm S D(F 2)$ & Mean $\pm S D$ (Total) & $\mathbf{p}$ \\
\hline \multirow[t]{3}{*}{ Male (F1) vs Female (F2) } & Time of retention (Q6) & $3.93 \pm 1.52$ & $4.23 \pm 1.35$ & $4.11 \pm 1.42$ & 0.101 \\
\hline & Orthodontist's opinion (Q8) & $4.63 \pm 0.80$ & $4.87 \pm 0.38$ & $4.78 \pm 0.60$ & $0.011^{*}$ \\
\hline & Periodontal status (Q9) & $4.03 \pm 0.87$ & $4.21 \pm 1.10$ & $4.14 \pm 1.02$ & 0.054 \\
\hline \multirow{4}{*}{$\leq 10$ years $(\mathrm{F} 1)$ vs $>10$ years $(\mathrm{F} 2)$} & Patient's demand (Q7) & $1.65 \pm 1.32$ & $1.17 \pm 1.09$ & $1.56 \pm 1.28$ & 0.065 \\
\hline & Orthodontist's opinion (Q8) & $4.79 \pm 0.61$ & $4.77 \pm 0.57$ & $4.78 \pm 0.60$ & 0.891 \\
\hline & Periodontal status (Q9) & $4.21 \pm 0.95$ & $3.87 \pm 1.25$ & $4.14 \pm 1.02$ & 0.190 \\
\hline & Need for a new restoration (Q10) & $4.05 \pm 1.07$ & $4.20 \pm 1.16$ & $4.08 \pm 1.08$ & 0.276 \\
\hline & Periodontal status (Q9) & $4.08 \pm 1.08$ & $4.20 \pm 0.97$ & $4.14 \pm 1.02$ & 0.517 \\
\hline & Need for a new restoration (Q10) & $3.96 \pm 1.12$ & $4.17 \pm 1.05$ & $4.08 \pm 1.08$ & 0.130 \\
\hline
\end{tabular}

\section{Oral hygiene recommendations}

\begin{abstract}
$100 \%$

\section{$80 \%$}

$60 \%$

$60 \%$
$20 \%$

$0 \%$

\section{$43.0 \%$}

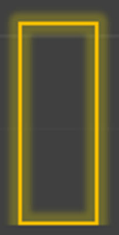

Same as a normal Interdental Brush patient

$82.5 \%$
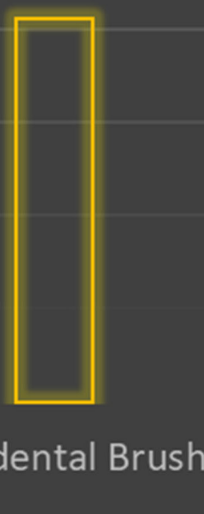

of $\leq 10$ years and those who worked at public clinics more frequently preferred to refer the patients to the orthodontist than the others $(p<0.05)$, but no associations were found in terms of the sex of the participants ( $p>0.05)$ (Figure 1). Participants with $\leq 10$ years of experience and those who worked at public clinics more frequently preferred to refer the patients who requested removal of the bonded retainer to the orthodontist who bonded the retainer previously $(p<0.05)$, but no association was found in terms of the sex of the participants ( $p>0.05$ ) (Figure 1).
\end{abstract}

$81.2 \%$

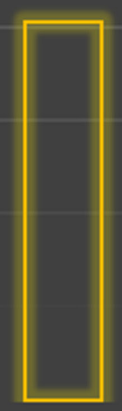

Dental Floss
$14.3 \%$

$0.6 \%$

Tooth pick

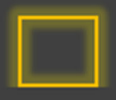

Others

Figure 2. Descriptive statistics revealing the rate of participants' oral hygiene recommendations

In terms of the factors affecting the choice to remove a bonded retainer, the most and the least importance were attribut- ed to the orthodontist's opinion (Mean \pm Standard deviation $=4.78 \pm 0.60$ ) and the patient's demand (Mean \pm Standard deviation=1.56 \pm 1.28 ), respectively. In terms of the time of retention and periodontal status, no significant differences were found among sexes, experience, or workplaces $(p>0.05)$. The participants who worked at private clinics attributed significantly more importance to the patient's demand than those who worked at public clinics $(p<0.001)$. No significant differences were observed among experience and sex factors in terms of the patient's demand ( $p>0.05$ ). The female participants attributed significantly more importance to the orthodontist's opinion than the male participants $(p=0.011)$. No significant differences 


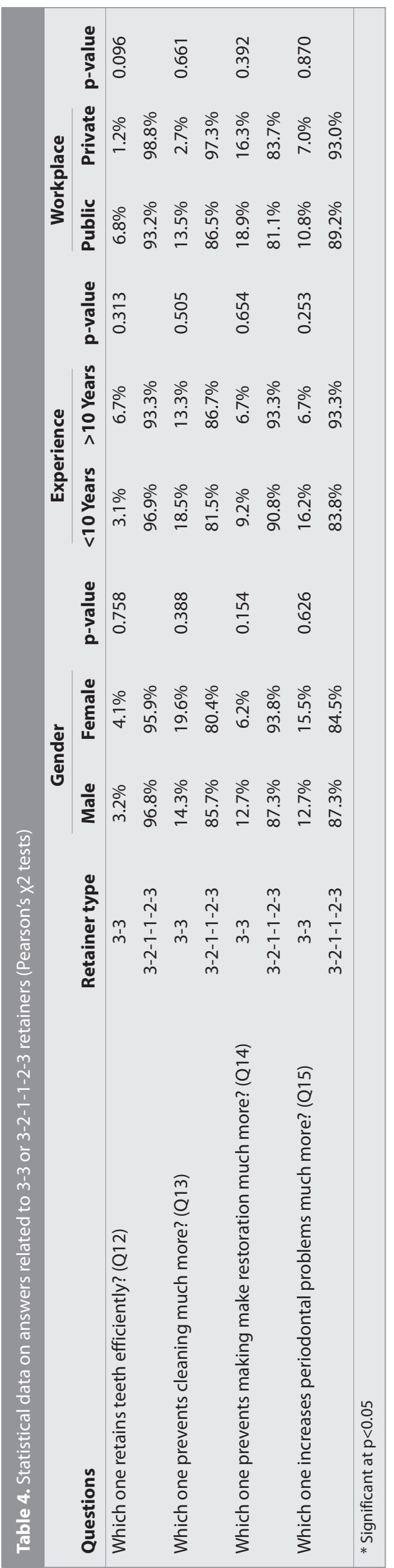

were obtained among the experience and workplace factors in terms of orthodontist's opinion ( $p>0.05)$. In terms of need of a new restoration, the female participants attributed significantly more importance than the male participants $(p=0.018)$. No significant differences were observed among the experience and workplace factors in terms of need of a new restoration $(p>0.05)$ (Table 3).

Most participants responded that 3-2-1-1-2-3 retainers retained teeth more efficiently compared to 3-3 retainers; however, these retainers prevent cleaning and making restoration, and increase the periodontal problems . No significant differences were obtained among the factors ( $p>0.05$ ) (Table 4).

Most participants recommended using interdental brush (82.5\%) and dental flosses (81.2\%) for oral hygiene of their patients, and toothpicks were the least recommended (0.6\%) (Figure 2).

\section{DISCUSSION}

In Turkey, the most commonly performed retainer procedures to prevent relapse after orthodontic treatments involve lingual retainers (10). Lingual retainers have been used by orthodontists for many years because they provide optimal retention in terms of function and esthetics. However, failures may occur in lingual retainers shortly after application (14). In retainer applications, the follow-up is performed by the orthodontists in the short term, whereas in the long term, such patients are usually referred to general dentists. However, the Turkish dentists, particularly those who worked at public institutions, stated that they preferred to refer the patient to an orthodontist when the retainer failed or when the patient demanded the retainer to be removed. There may be several possible reasons for this situation. Although dentists are allowed to charge for certain state-specified treatments in public services, they cannot charge for some treatments such as retainer bonding. Besides, in Turkey, the workload in the public sector is much higher than that in private clinics (18). Dentists working at private clinics tended to remove the retainer more often when patients demanded the removal of a bonded retainer. This trend may be related to seeking provision of patient satisfaction and confidence.

Ideally, the follow-up examination of the orthodontic patients should be performed by the orthodontist. However, lengthy recommended retention periods (often, the retention period may be for lifetime) may necessitate the orthodontists to share the responsibility of these patients with dentists. In some countries, it is considered that this responsibility belongs to general dentists 6 months after the application of the retainer (16). However, in Turkey, this responsibility is generally given to orthodontists. In our study, in general, inexperienced practitioners did not dare to repair debonded or broken lingual retainers. The reason may be that, over the years, experienced practitioners improve their skills in dentistry practice by treating more patients and participating in courses. However, in a study conducted in Switzerland, unlike the case in our study, most dentists preferred rebonding in the case of retainer failures. In addition, when the patient demanded the retainer removal, they preferred to inform them about the possible consequences and leave the retainer in situ (16). 
While deciding to remove a bonded retainer, the dentists attributed more importance to the orthodontist's opinion than to the patient's demand. Interestingly, time of retention and patient's demand in Switzerland were more important than the orthodontist's opinion (16). Dentists who worked at private clinics more frequently considered patient demand more important than orthodontist's opinion in preferring to remove the retainer. Physicians who work at private clinics might not be able to refuse patients' demands in order not to lose income. Furthermore, the female participants attributed more importance to the orthodontist's opinion, but participants of both sexes seemed to attach great importance to the opinion of the orthodontist. Retainers may need to be removed when a new restoration is needed based on the position of the dental caries. In these cases, surprisingly, it seemed that female practitioners tended to remove the bonded retainer more often. However, it was difficult to predict which factors played a role in these differences between sexes.

Although bonded retainers are as effective as removable ones, in the long term, it was shown that they can escalate periodontal problems, plaque formation, gingival recession, and calculus accumulation $(19,20)$. In general, lingual retainers may be bonded as 3-3 or 3-2-1-1-2-3. Although the Turkish dentists considered the 3-2-1-1-2-3 retainers to be better, they thought that these retainers had some disadvantages in terms of periodontal problems and restoration on the related teeth. In fact, which retainer is ideal is a controversial issue, and the periodontal status of the patient, the amount of mobility of the teeth, and the type of malocclusion are the factors that may affect this choice (21). Renkema et al. (22) found that the failure rate of lower retainers bonded to all the anterior teeth was higher than those that were bonded to only the canines. However, in patients who have teeth with spacing and extreme rotations in the anterior region, the $3-2-1-1-2-3$ retainers may be the right choice for maintaining stability. The 3-3 retainers should be considered in cases of periodontal problems (5). Many researchers claimed that the 3-3 retainers were easier to be cleaned by the patients and dentists $(16,21)$. It seems that the approaches of the Turkish participants to these retainers were in agreement with the results of these studies. Additionally, in our study, there was no significant difference between the participants in terms of the answers given to the questions about retainer types.

Insufficient oral hygiene in orthodontic patients with lingual retainers is an important factor in the development of white spot lesions, dental caries, and gingival inflammation due to the dental plaque accumulation (23). Tooth brushing is considered the primary means of plaque reduction to prevent dental caries or gingival inflammation (24). However, excess plaque deposition in areas around the lingual retainer require patients to use hygiene tools, such as an interdental brush and dental floss in addition to tooth brushing (23). In this respect, as in Switzerland, Turkish dentists highly recommend interdental brush and dental floss to the patients with lingual retainers $(16,25)$. However, in Netherlands, the use of a toothpick was recommended rather than interdental brush and dental floss (5).

\section{CONCLUSION}

When all these data were reviewed comprehensively, it was observed that Turkish dentists preferred a conservative approach in the cases of lingual retainer failure and generally referred the patients to the orthodontists. Besides, it was observed that different demographic characteristics had an impact on the approaches of dental practitioners.

Ethics Committee Approval: This study was approved by Ethics committee of Sutcu Imam University, (Approval No: 2019-66).

Informed Consent: Written informed consent was obtained from the participants who agreed to take part in the study.

Peer-review: Externally peer-reviewed.

Author Contributions: Supervision - A.K.; Design - Ö.H, A.K.; Supervision - A.K.; Resources - A.K.; Materials - A.K.; Data Collection and/or Processing - Ö.H.; Analysis and/or Interpretation - Ö.H.; Literature Search - Ö.H.; Writing Manuscript - Ö.H, A.K.; Critical Review - A.K.

Conflict of Interest: The authors have no conflict of interest to declare.

Financial Disclosure: The authors declared that this study has received no financial support.

\section{REFERENCES}

1. Blake M, Bibby K. Retention and stability: A review of the literature. Am J Orthod Dentofacial Orthop 1998; 114: 299-306. [Crossref]

2. Littlewood S, Millett D, Doubleday B, Bearn D, Worthington $H$, Sampson WJ. Retention procedures for stabilizing tooth position after treatment with orthodontic braces. Aust Dent J 2006; 51: 94-5. [Crossref]

3. Blake M, Garvey MT. Rationale for retention following orthodontic treatment. J Can Dent Assoc 1998; 64: 640-3.

4. Padmos JAD, Fudalej PS, Renkema AM. Epidemiologic study of orthodontic retention procedures. Am J Orthod Dentofacial Orthop 2018; 153: 496-504. [Crossref]

5. Renkema AM, Hélène Sips ET, Bronkhorst E, Kuijpers-Jagtman AM. A survey on orthodontic retention procedures in The Netherlands. Eur J Orthod 2009; 31: 432-7. [Crossref]

6. Singh P, Grammati S, Kirschen R. Orthodontic retention patterns in the United Kingdom. J Orthod 2009; 36: 115-21. [Crossref]

7. Valiathan M, Hughes E. Results of a survey-based study to identify common retention practices in the United States. Am J Orthod Dentofacial Orthop 2010; 137: 170-7. [Crossref]

8. Wong PM, Freer TJ. A comprehensive survey of retention procedures in Australia and New Zealand. Aust Orthod J 2004; 20: 99-106.

9. Vandevska-Radunovic V, Espeland L, Stenvik A. Retention: Type, duration and need for common guidelines. A survey of Norwegian orthodontists. Orthodontics (Chic) 2013; 14: 2-9. [Crossref]

10. Paşaoğlu A, Aras I, Mert A, Aras A. Survey on retention protocols among Turkish orthodontists. Turk J Orthod. 2016; 29:51-8. [Crossref]

11. Watted N, Wieber M, Teuscher T, Schmitz N. Comparison of incisor mobility after insertion of canine-to-canine lingual retainers bonded to two or to six teeth a clinical study. J Orofac Orthop 2001; 62: 387-96. [Crossref]

12. Butler J, Dowling P. Orthodontic bonded retainers. J Ir Dent Assoc 2005; 51: 29-32.

13. Pandis N, Christensen L, Eliades T. Long-term clinical failure rate of molar tubes bonded with a self-etching primer. Angle Orthod 2005; 75: $1000-2$.

14. Taner T, Aksu M. A prospective clinical evaluation of mandibular lingual retainer survival. Eur J Orthod 2012; 34: 470-4. [Crossref] 
15. Schneider E, Ruf S. Upper bonded retainers: Survival and failure rates. Angle Orthod 2011; 81: 1050-6. [Crossref]

16. Habegger M, Renkema AM, Bronkhorst E, Fudalej PS, Katsaros C. A survey of general dentists regarding orthodontic retention procedures. Eur J Orthod 2017; 39: 69-75. [Crossref]

17. Turkish statistical institute. Statistical data in Turkey internet. 2018. Available from: http://www.tuik.gov.tr.

18. Hatipoglu O, Temur KT. The approaches of Turkish dentists to the invasive treatment. Int Dent Res 2018; 8: 56-62. [Crossref]

19. Rody WJ, Jr., Akhlaghi H, Akyalcin S, Wiltshire WA, Wijegunasinghe M, Filho GN. Impact of orthodontic retainers on periodontal health status assessed by biomarkers in gingival crevicular fluid. Angle Orthod. 2011; 81: 1083-9. [Crossref]

20. Levin L, Samorodnitzky-Naveh GR, Machtei EE. The association of orthodontic treatment and fixed retainers with gingival health. J Periodontol. 2008; 79: 2087-92. [Crossref]
21. Wouters C, Lamberts TA, Kuijpers-Jagtman AM, Renkema AM. Development of a clinical practice guideline for orthodontic retention. Orthod Craniofac Res. 2019; 22: 69-80 [Crossref]

22. Renkema A-M, Renkema A, Bronkhorst E, Katsaros C. Long-term effectiveness of canine-to-canine bonded flexible spiral wire lingual retainers. Am J Orthod Dentofacial Orthop 2011; 139: 614-21. [Crossref]

23. Chowdhary S, Jain RK, Santhosh Kumar M. Comparison of oral hygiene and gingival health response in patients wearing two types of orthodontic retainers. Drug Invent Today 2018; 10: 1790-2.

24. Van der Weijden FA, Slot DE. Efficacy of homecare regimens for mechanical plaque removal in managing gingivitis a meta review. J Clin Periodontol 2015; 42: 77-91. [Crossref]

25. Lai CS, Grossen JM, Renkema AM, Bronkhorst E, Fudalej PS, Katsaros C. Orthodontic retention procedures in Switzerland. Swiss Dent J 2014; 124: 655-61. 